\section{Breathing life into lung models}

\section{By Michael J. Haas, Senior Writer}

A team of Boston researchers has developed a microfluidics device that mimics key lung functions_-including breathing_and can reproduce the effects of inhaled substances on the lung's alveolar-capillary interface better than existing in vitro models. ${ }^{1}$ The team thinks the lung-on-a-chip technology could be used to screen inhaled drug candidates for toxicity and efficacy more reliably than other in vitro models.

Current in vitro models of the lung, such as transwell systems, can reproduce tissue interfaces in the organ-such as that between alveoli and vascular endothelium - but not the mechanical processes of physiological breathing. They also cannot show how the stresses of breathing affect lung cell responses to inhaled substances.

To overcome these drawbacks, a team led by Donald Ingber designed and constructed a microfluidics system that reproduces both the lung's alveolar-capillary interface and the mechanical effects of breathing on that interface-all on a polymer chip about $2 \mathrm{~cm}$ long. Ingber is professor of pathology at Harvard Medical School and Children's Hospital Boston, professor of bioengineering at Harvard University and director of Harvard's Wyss Institute for Biologically Inspired Engineering.

The alveolar-capillary interface on the chip consists of two microchannels-one containing human alveolar epithelial cells and a layer of air and the other containing human pulmonary microvascular endothelial cells and a flowing layer of culture medium. The channels are separated by a porous, $10 \mu \mathrm{m}$-thick membrane of flexible polymer coated with extracellular matrix.

The interface between alveolar and microvascular cells is flanked by two additional chambers separated from it by thin walls. Upon application of a vacuum to the side chambers, the thin walls deform and cause the polymer membrane to stretch, thus mimicking the effects of breathing (see Figure 1, "Lung on a chip").

As a test of the device, the team introduced the proinflammatory cytokine tumor necrosis factor- $\alpha$ (TNF- $\alpha$ ) or Escherichia coli into the interface and showed that the resulting inflammatory responses to each closely resembled those seen in the lungs of animals.

Ingber's group also used the device to study the effects of breathing on the alveolar-capillary interface's response to silica nanoparticles and carboxylated quantum dots. The interface exhibited greater uptake of these particle types and more pronounced inflammatory and toxic oxidative responses to them when vacuum was applied to the side chambers than when there was no breath-mimicking vacuum or when the transwell system was used. By contrast, other nanomaterials such as carbon nanotubes and gold nanoparticles did not induce inflammatory or toxic responses, even with breath-mimicking vacuum.

These experiments demonstrated that the chip can model materialspecific responses to nanoparticle-based therapeutics, Ingber told SciBX.

"This approach is really a breakthrough toward the holy grail of eliminating the need to use animal models to get reproducible and meaningful results," said Robert Clarke, VP of research at pulmonary infection company Pulmatrix Inc. "It has taken lung modeling to the next level by adding the mechanical stress of breathing."

Paul Rennert, principal scientist of molecular discovery in Biogen Idec Inc.'s Department of Immunobiology, agreed. “This is a fascinating technical advance - a real tour de force - that appears to offer immediate advantages over traditional models" such as its ease of use, response to multiple stimuli and ability to mimic the tissue stretching caused by breathing.

\section{Expanding applications}

Clarke and Rennert envisioned multiple uses for the chip described in Science. They added that the technology's applications would be even broader if the interface incorporated immune cells and/or other lung cell types and tissue interfaces.

Rennert noted that Ingber's team introduced neutrophils into the chip and monitored migration of the cells through and across the interface. "I'd like to see similar work with activated lymphocytes, macrophages, dendritic cells and granulocytes," he said.

Clarke agreed that including immune cells in addition to neutrophils would help determine whether the interface "truly mimics the cell-to-cell cross-talk that occurs in the airway." He also wanted to know whether the chip could model the upper airway interface between epithelial cells and endothelial or smooth muscle cells.

"That would be interesting to us because we're looking at infections in the upper airway" and how asthma and chronic obstructive pulmonary disease (COPD) exacerbate those infections, he said.

Ingber said the team does plan to incorporate other immune cells into the chip.

He added that the upper airway tissue interface could be modeled on a chip as Clarke proposed, "but we have not pursued this yet."

Even with only two cell types, Clarke said he could think of several ways Pulmatrix could use the technology in its drug development programs, such as studying how proteins or other large, inhaled molecules cross the alveolar-capillary interface and screening first-in-class drug therapies.

"I would be interested in experiments that looked at how our iCALM product affects chemokine and cytokine production in the interface and how iCALM affects cellular responses to drugs or viruses introduced in the system," he said.

Pulmatrix's lead compound, PUR003, is an inhaled cationic airway lining modulator (iCALM) that creates a barrier at the lining of the airway against viruses in the respiratory tract. The compound is in Phase I/II testing to treat and prevent influenza infection. 
Figure 1. Lung on a chip. Researchers have developed a $2 \mathrm{~cm}-$ long polymer device that mimics the function of the human lung. [a] The microfluidics system incorporates an alveolar-capillary interface that is flanked by two side chambers.

[b] The alveolar-capillary interface consists of a porous, flexible, $10 \mu \mathrm{m}$-thick polymer membrane coated with extracellular matrix (ECM) that separates a channel containing human alveolar epithelial cells and a layer of air from a channel containing human pulmonary microvascular endothelial cells and a flowing layer of cell culture media.

[c] Application of vacuum to the side chambers deforms the thin walls separating those chambers from the interface, causing the flexible polymer membrane to stretch-thus mimicking the mechanical effects of breathing.

Rennert thinks the most immediate use of the chip is fast toxicology screens of compound classes with known side effects on the lung-such as agonists of sphingoside-1-phosphate receptor 1 (S1PR1; S1P1; EDG1), a protein that plays a role in pulmonary intercellular adhesion. Multiple S1PR1 agonists are in development to treat multiple sclerosis (MS), psoriasis and cancer.

\section{Chips on the table}

Ingber said his team is seeking industry partners in pharmacology and toxicology to identify diseases for which animal models are unavailable or ineffective at predicting results in humans.

"For example, we believe that our technology would have particular relevance for screening aerosol-based drugs or nanotherapeutics in terms of maximizing absorption and minimizing the inflammatory and toxic side effects," he said.

The team also is developing microdevices that mimic other organssuch as the beating heart and peristaltic gut_-in which mechanical stresses likely affect absorption, bioavailability and toxicity of drug compounds.

"We are also exploring a cancer-on-a-chip model," Ingber said.

Children's Hospital Boston has applied for a patent covering both the findings reported in Science and other organ-on-a-chip technologies. The IP is available for licensing through Harvard's Wyss Institute, Ingber said.

Haas, M.J. SciBX 3(28); doi:10.1038/scibx.2010.847

Published online July 22, 2010

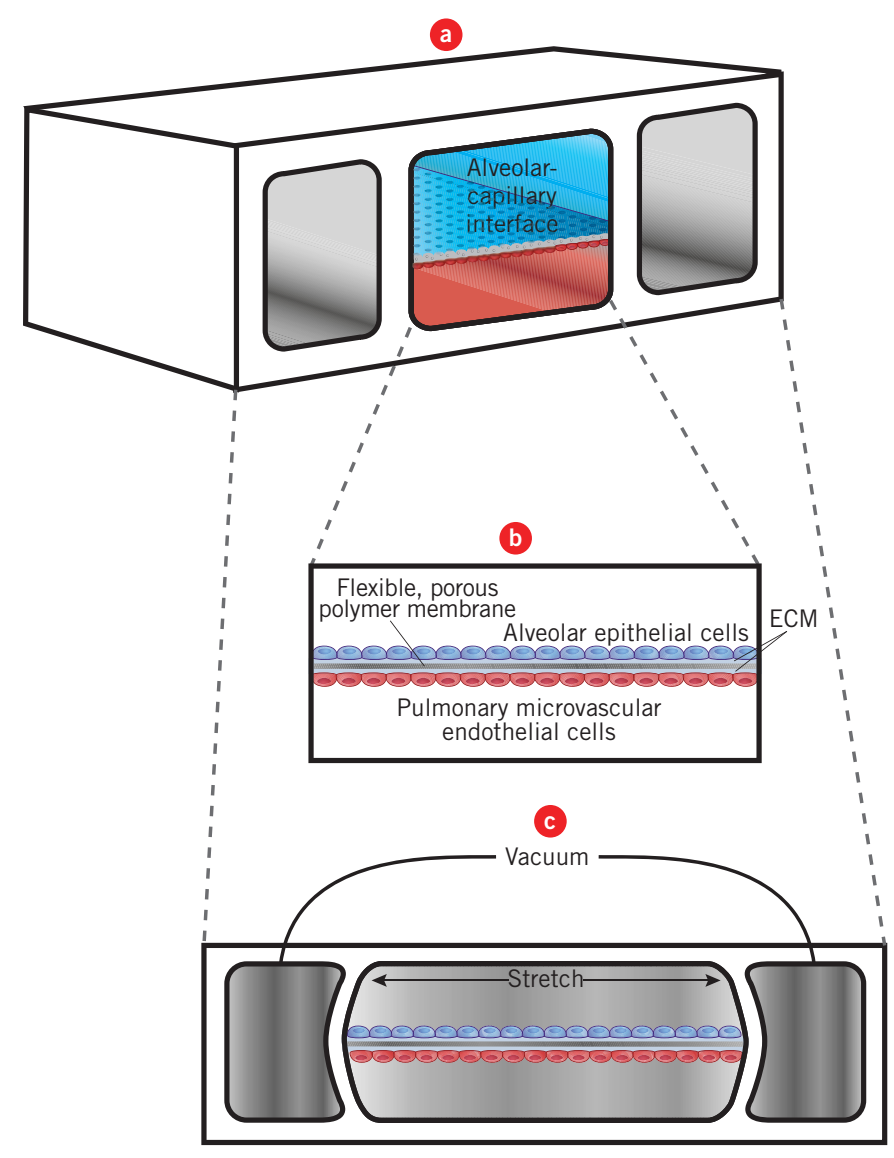

REFERENCES

1. Huh, D. et al. Science; published online June 24, 2010; doi:10.1126/science.1188302

Contact: Donald E. Ingber, Harvard University, Boston, Mass. e-mail: don.ingber@wyss.harvard.edu Contact: Dan Huh, same affiliation as above e-mail: dan.huh@wyss.harvard.edu

COMPANIES AND INSTITUTIONS MENTIONED

Biogen Idec Inc. (NASDAQ:BIIB), Weston, Mass. Children's Hospital Boston, Boston, Mass.

Harvard Medical School, Boston, Mass.

Harvard University, Boston, Mass.

Pulmatrix Inc., Lexington, Mass. 